MINIREVIEW

\title{
Nitric oxide: orchestrating hypoxia regulation through mitochondrial respiration and the endoplasmic reticulum stress response
}

\author{
Weiming XU, Ian G. CHARLES, Salvador MONCADA* \\ Wolfson Institute for Biomedical Research, University College London, The Cruciform Building, Gower Street, London, WC1E \\ $6 B T, U K$
}

\begin{abstract}
Mitochondria have long been considered to be the powerhouse of the living cell, generating energy in the form of the molecule ATP via the process of oxidative phosphorylation. In the past 20 years, it has been recognised that they also play an important role in the implementation of apoptosis, or programmed cell death. More recently it has become evident that mitochondria also participate in the orchestration of cellular defence responses. At physiological concentrations, the gaseous molecule nitric oxide (NO) inhibits the mitochondrial enzyme cytochrome c oxidase (complex IV) in competition with oxygen. This interaction underlies the mitochondrial actions of NO, which range from the physiological regulation of cell respiration, through mitochondrial signalling, to the development of "metabolic hypoxia" - a situation in which, although oxygen is available, the cell is unable to utilise it.
\end{abstract}

Keywords: nitric oxide, hypoxia, mitochondria, endoplasmic reticulum, stress.

\section{NO AND MITOCHONDRIAL RESPIRATION}

Nitric oxide (NO) plays an important role as an interand intra-cellular messenger, acting in the cardiovascular system, in the nervous system and as a component of the immune system [1]. NO is a diatomic free radical which is a gas at room temperature, making it highly diffusible within the vasculature. One of its major targets is the soluble guanylyl cyclase (sGC). The binding of NO to $\mathrm{Fe}^{2+}$ in the haem group of sGC activates the enzyme and increases the concentration of cGMP (guanosine 3', 5'cyclic monophosphate). The NO/cGMP signalling pathway has been demonstrated to be important in many biological systems including blood pressure regulation, platelet aggregability, smooth muscle relaxation and peripheral and central neurotransmission.

Recent work has shown that NO, in the nanomolar concentration range that activates $\mathrm{SGC}$, can also bind to cytochrome c oxidase (complex IV) and inhibit it in a manner that is reversible and in competition with oxygen $[2,3]$. Cytochrome c oxidase is the terminal enzyme of the mitochondrial respiratory chain and is responsible for

\footnotetext{
*Correspondence: Salvador MONCADA

Fax: +44-0-2072090470

E-mail: s.moncada@ucl.ac.uk
}

$90 \%$ of cellular oxygen consumption in mammals. The enzyme catalyses the oxidation of cytochrome $\mathrm{c}^{2+}$ to cytochrome $\mathrm{c}^{3+}$ and the reduction of oxygen to water, which is coupled to the pumping of protons across the inner mitochondrial membrane. Because NO competes with oxygen at the cytochrome c oxidase, increases in NO concentration can prevent the enzyme from using any available oxygen, thus causing a kind of "metabolic hypoxia" [4].

Such "metabolic hypoxia" has profound biological consequences, particularly with regard to the regulation of the hypoxia-inducible factor (HIF)-1. HIF-1 is a transcription factor that plays a major role in the response of tissues to low partial pressures of oxygen, by upregulating genes such as those involved in glycolysis and angiogenesis [5]. At normal oxygen concentrations HIF-1 is regulated in an oxygen_dependent manner by a family of prolyl hydroxylases that prevent stabilisation of HIF- $1 \alpha$ its subsequent dimerisation with HIF- $1 \beta$ and the consequent activation of target genes. At low oxygen concentrations (1-3\%) prolyl hydroxylase activity is inhibited, and HIF-1 $\alpha$ accumulates, resulting in the activation of target gene expression. However, if cells maintained at $1-3 \%$ oxygen are exposed to low concentrations of NO, stabilisation of HIF- $1 \alpha$ is prevented [6]. We have shown that, in hypoxic conditions, $\mathrm{NO}$ and other inhibitors of mitochondrial respiration can cause redistribution of oxygen away from the electron trans- 
port chain towards non-respiratory oxygen-dependent targets such as prolyl hydroxylases [7]. Since more than 70 genes (including those involved in erythropoiesis, angiogenesis and glycolysis) are known to be regulated at the transcriptional level by HIF-1 [5], NO-mediated inhibition of respiration may profoundly modify the signalling consequences of hypoxia.

\section{NO AND THE ENDOPLASMIC RETICULUM STRESS RESPONSE}

Until recently, the main function of mitochondria was considered to be the production of energy; however mitochondria can also play an important role in intracellular signalling, for example by coupling calcium signalling with the endoplasmic reticulum (ER) [8-10]. Studies using genetically targeted $\mathrm{Ca}^{2+}$-selective probes (such as the bioluminescent protein aequorin) or fluorescent dyes (such as rhod-2) have provided a direct and unambiguous readout of mitochondrial $\mathrm{Ca}^{2+}$ changes in living cells following receptor stimulation. Recently we found that in NOgenerating cells there is a disruption in the respiratory chain and a decreased respiration rate that is accompanied by a mitochondrial $\mathrm{Ca}^{2+}$ flux [11]. These findings are in agreement with previous reports showing that exogenous NO $[12]$ and peroxynitrite $[13,14]$ can result in the release of $\mathrm{Ca}^{2+}$ from mitochondria. Interestingly, Nowicky and Duchen reported changes in $\left[\mathrm{Ca}^{2+}\right]_{i}$ and membrane currents when mitochondrial respiration in dissociated rat hippocampal neurons was impaired by inhibition of cytochrome c oxidase using cyanide, or by hypoxia [15]. It is possible that disrupting electron transfer at cytochrome $\mathrm{c}$ oxidase may result in ionic readjustment and modulation of the $\mathrm{Ca}^{2+}$ flux between the mitochondria and the ER. We found that this NO-mediated change in $\mathrm{Ca}^{2+}$ flux is sufficient to activate the $\mathrm{Ca}^{2+}$-dependent protease (site-1 protease, S1P) involved in a regulated intermembrane proteolysis (RIP) pathway. S1P, in association with the site2 protease located in the Golgi apparatus, cleaves the ER stress-regulated transmembrane transcription factor $\mathrm{p} 90$ ATF6. The resulting soluble transcription factor p50 ATF6 is then free to translocate to the nucleus where it subsequently activates ER stress-responsive genes, such as glucose regulated protein 78 (Grp78) [11].

Grp78 is a $\mathrm{Ca}^{2+}$-binding chaperone protein with cytoprotective properties. It can be up-regulated by hypoxic conditions and by glucose starvation. Grp78 associates transiently with nascent proteins, facilitating their translocation into the ER and aiding in their folding and transport through the ER. Grp78 appears to be a master switch of the unfolded protein response (UPR) and ER stress. We found that the NO-mediated up-regulation of Grp78 provides significant cytoprotection against toxic agents including thapsigargin (a selective $\mathrm{ER} \mathrm{Ca}^{2+}$-ATPase inhibitor). This cytoprotection is abolished following treatment with cyclosporin A (CsA), which prevents mitochondrial $\mathrm{Ca}^{2+}$ efflux, and BAPTA-AM, an intracellular chelator. The NO-mediated ER stress response is diminished in $r h o^{\circ}$ cells that are devoid of mitochondrial DNA. Therefore, our work has shown that NO signals, via mitochondrial respiration, to the ER stress response with consequences for cell survival or death [11].

Apart from Grp78, a number of other genes have been shown to be regulated by the $\mathrm{Ca}^{2+}$-dependent serine protease S1P. These include genes for growth factors, transcription factors and viral glycoproteins that are involved in neurological, cardiovascular and immunological systems. Our findings suggest that the NO-mediated $\mathrm{Ca}^{2+}$ signalling pathway may well be important in their regulation.

\section{CONCLUSION}

The coupling of mitochondrial signalling and the ER is an emerging area of interest, and a common apoptosis pathway has recently been described in BAX and BAK regulation in which the ER and mitochondria are coupled via $\mathrm{Ca}^{2+}[16]$. It will be important to explore further how the NO-mediated ER signalling we have described interacts with BAX and BAK pathways to regulate mitochondria-mediated apoptosis.

\section{REFERENCES}

1 Moncada S, Higgs A. The L-arginine-nitric oxide pathway. New Engl J Med 1993; 329:2002-12.

2 Brown GC. Regulation of mitochondrial respiration by nitric oxide inhibition of cytochrome c oxidase. Biochim Biophys Acta 2001; 1504:46-57.

3 Cooper CE. Nitric oxide and cytochrome oxidase: substrate, inhibitor or effector? Trends Biochem Sci 2002; 27:33-9.

4 Moncada S, Erusalimsky JD. Does nitric oxide modulate mitochondrial energy generation and apoptosis? Nat Rev Mol Biol 2002; 3:214-20.

5 Semenza GL. Signal transduction to hypoxia-inducible factor. Biochem Pharmacol 2002; 64:993-8.

6 Mateo J, Garcia-Lecea M, Cadenas S, Hernandez C, Moncada S. Regulation of hypoxia-inducible factor-1 alpha by nitric oxide through mitochondria-dependent and -independent pathways. Biochem J 2003; 376:537-44.

7 Hagen T, Taylor CT, Lam F, Moncada S. Redistribution of intracellular oxygen in hypoxia by nitric oxide: effect on HIF1alpha. Science 2003; 302:1975-8.

8 Rizzuto R, Pinton P, Carrington W, et al. Close contacts with the endoplasmic reticulum as determinants of mitochondrial $\mathrm{Ca}^{2+}$ responses. Science 1998; 280:1763-6.

9 Rizzuto R, Duchen M, Pozzan T. Flirting in little space: The $\mathrm{ER} /$ mitochondria $\mathrm{Ca}^{2+}$ liaison. Sci STKE 2004; (215):re1.

10 Carafoli, E. Historical review: mitochondria and calcium: Ups and downs of the unusual relationship. Trends Biochem Sci 2003; 28:175-81 
$11 \mathrm{Xu} \mathrm{W}$, Liu L, Charles IG, Moncada S. Nitric oxide induces coupling of mitochondrial signalling with the endoplasmic reticulum stress response. Nat Cell Biol 2004; 6:1129-34.

12 Horn TF, Wolf G, Duffy S, et al. Nitric oxide promotes intracellular calcium release from mitochondria in striatal neurons. FASEB J 2002; 16:1611-22.

13 Packer MA, Murphy MP. Peroxynitrite causes calcium efflux from mitochondria which is prevented by cyclosporin A. FEBS Lett 1994; 345:237-40.
14 Schweizer M, Richter C. Peroxynitrite stimulates the pyridine nucleotide-linked $\mathrm{Ca}^{2+}$ release from intact rat liver mitochondria. Biochemistry 1996; 35:4524-8.

15 Nowicky AV, Duchen MR. Changes in $\left[\mathrm{Ca}^{2+}\right] \mathrm{i}$ and membrane currents during impaired mitochondrial metabolism in dissociated rat hippocampal neurons. J Physiol 1998; 507:131-45.

16 Scorrano L, Oakes SA, Opferman JT, et al. BAX and BAK regulation of endoplasmic reticulum $\mathrm{Ca}^{2+}$ : a control point for apoptosis. Science 2003; 300:135-9. 